\title{
Does polynomial parallel volume imply convexity?
}

\section{Matthias Heveling • Daniel Hug • Günter Last}

Received: 1 April 2003 / Revised version: 16 July 2003 /

Published online: 6 January 2004 - (C) Springer-Verlag 2004

\begin{abstract}
For a non-empty compact set $A \subset \mathbb{R}^{d}, d \geq 2$, and $r \geq 0$, let $A_{\oplus r}$ denote the set of points whose distance from $A$ is $r$ at the most. It is well-known that the volume, $V_{d}\left(A_{\oplus r}\right)$, of $A_{\oplus r}$ is a polynomial of degree $d$ in the parameter $r$ if $A$ is convex. We pursue the reverse question and ask whether $A$ is necessarily convex if $V_{d}\left(A_{\oplus r}\right)$ is a polynomial in $r$. An affirmative answer is given in dimension $d=2$, counterexamples are provided for $d \geq 3$. A positive resolution of the question in all dimensions is obtained if the assumption of a polynomial parallel volume is strengthened to the validity of a (polynomial) local Steiner formula.
\end{abstract}

Mathematics Subject Classification (2000): 52A38, 28A75, 52A22, 53C65

\section{Introduction and results}

Convex sets in a Euclidean space can be characterized in various ways within the class of closed sets. Among the classical characteristic properties are the existence of supporting hyperplanes through each boundary point and the existence of a nearest point map. The present paper investigates an apparently new characterization of the convexity of a closed set, in terms of the volumes of its (local) parallel sets.

Let $A$ denote a non-empty compact subset of $\mathbb{R}^{d}, d \geq 2$, and let $A_{\oplus r}$ be the parallel set of $A$ at distance $r \geq 0$, i.e. the set of all points $x \in \mathbb{R}^{d}$ whose distance from $A$ is at most $r$. Further, let $V_{d}$ denote the volume function (Lebesgue measure). In the following, we consider the parallel volume, $V_{d}\left(A_{\oplus r}\right)$, of $A$ as a function of the distance parameter $r \geq 0$. If $A$ is convex, then

\section{HEVELING}

Institut für Mathematische Stochastik und Mathematisches Institut II, Universität Karlsruhe (TH), Englerstr. 2, D-76128 Karlsruhe, Germany

(e-mail: matthias.heveling@math.uni-karlsruhe.de)

D. HuG

Mathematisches Institut, Albert-Ludwigs-Universität Freiburg, Eckerstr. 1, D-79104 Freiburg, Germany (e-mail: daniel.hug@math.uni-freiburg.de)

G. LAST

Institut für Mathematische Stochastik, Universität Karlsruhe (TH), Englerstr. 2, D-76128

Karlsruhe, Germany (e-mail: g. last@math.uni-karlsruhe.de) 


$$
V_{d}\left(A_{\oplus r}\right)=\sum_{j=0}^{d} r^{d-j} \kappa_{d-j} V_{j}(A),
$$

where $\kappa_{j}$ is the ( $j$-dimensional) volume of the Euclidean unit ball in $\mathbb{R}^{j}$ and the coefficients $V_{0}(A), \ldots, V_{d}(A)$ are the intrinsic volumes of the convex body $A$ (see, e.g., [16, (4.2.27)]). It is well known that $V_{d-1}(A)$ is half the surface area (if $A$ has non-empty interior) and $V_{0}(A)=1$. The Steiner formula (1.1) and its ramifications are of central importance in geometry. Applications of the fundamental relation (1.1) and of the functionals $V_{j}$ defined by it can be found in various other branches of mathematics including stochastic geometry [18], [17], statistics [9], [11], discrete mathematics [2], geometric functional analysis [7], and recently also in physics [12], [13].

It is natural to investigate conditions under which a Steiner formula of the form (1.1) can be obtained for sets which are not necessarily convex. Results in this direction, which are available in the literature, usually state a Steiner formula under regularity assumptions on the set and for a restricted range of the distance parameter $r$; see, for instance, the classical contributions by Hadwiger [8] and Federer [4]. In the present paper, we reverse this point of view and ask the following question.

\section{Does polynomial parallel volume imply convexity?}

It is tempting to guess a positive answer to this question. Simple examples (see Section 4), however, show that, for all dimensions $d \geq 3$, there are non-convex sets whose parallel volume is a polynomial. On the other hand, in dimension $d=2$ we can answer our question in an affirmative way.

Theorem 1. Let $A \subset \mathbb{R}^{2}$ be compact such that

$$
V_{2}\left(A_{\oplus t}\right)=V_{2}(A)+c_{1} t+c_{2} t^{2}, \quad t \geq 0,
$$

for some $c_{1}, c_{2} \in \mathbb{R}$. Then $A$ is convex.

Note that the seemingly more general assumption that $V_{2}\left(A_{\oplus t}\right)$ is a polynomial of degree $n \in \mathbb{N}$ in the parameter $t \geq 0$ immediately implies (1.2). Theorem 1 provides a characterization of convexity by a global property. A corresponding result is wrong in higher dimensions, but the conclusion of Theorem 1 remains true under a stronger (local) hypothesis. We prepare such a result, together with an extension, by introducing some more notation.

Let $A$ denote an arbitrary non-empty closed subset of $\mathbb{R}^{d}$. The distance $d(A, z)$ from a point $z \in \mathbb{R}^{d}$ to a set $A \subset \mathbb{R}^{d}$ is defined as $\inf \{|y-z|: y \in A\}$, where $|\cdot|$ denotes the Euclidean norm on $\mathbb{R}^{d}$ and $\inf \emptyset:=\infty$. We put $p(A, z):=y$ whenever $y$ is a uniquely determined point in $A$ with $d(A, z)=|y-z|$, and we call this point the metric projection of $z$ on $A$. If $0<d(A, z)<\infty$ and $p(A, z)$ is defined, then $p(A, z)$ lies on the boundary $\partial A$ of $A$ and we put $u(A, z):=$ 
$(z-p(A, z)) / d(A, z)$. The exoskeleton $\operatorname{exo}(A)$ of $A$ consists of all points of $\mathbb{R}^{d} \backslash A$ which do not admit a metric projection on $A$. This is a measurable set (see, e.g., Lemma 6.1 in [10]) satisfying $\mathcal{H}^{d}(\operatorname{exo}(A))=0$, where $\mathcal{H}^{d}$ denotes the $d$-dimensional Hausdorff measure. Clearly, if $A$ is convex, then $\operatorname{exo}(A)=\emptyset$. We extend the definition of $p(A, z) \in \mathbb{R}^{d}$ and $u(A, z) \in S^{d-1}$ in a suitable and measurable way to all $z \in \mathbb{R}^{d}$. Here $S^{d-1}$ denotes the unit sphere $\left\{z \in \mathbb{R}^{d}:|z|=1\right\}$.

Now let $A \subset \mathbb{R}^{d}$ be a non-empty closed convex set. Extending and unifying results by Aleksandrov [1], Fenchel \& Jessen [6] and Federer [4], Schneider [14], [15] has shown that $A$ satisfies a local Steiner formula. It states that, for any measurable bounded function $f: \mathbb{R}^{d} \times S^{d-1} \rightarrow \mathbb{R}$ with compact support and $t \geq 0$,

$$
\begin{array}{rl}
\int_{\mathbb{R}^{d} \backslash A} & f(p(A, z), u(A, z)) \mathbf{1}\{d(A, z) \leq t\} d z \\
= & \sum_{j=0}^{d-1} t^{d-j} \kappa_{d-j} \int f(x, u) C_{j}(A ; d(x, u)),
\end{array}
$$

where $d z$ denotes integration with respect to Lebesgue measure. The measures $C_{j}(A ; \cdot), j \in\{0, \ldots, d-1\}$, are the support measures (generalized curvature measures) of the convex set $A$, which simultaneously generalize the surface area measures and the curvature measures of $A$. They are non-negative, locally finite and concentrated on $\partial A \times S^{d-1}$. We refer to [16] for further background information.

We now modify our initial question as follows:

Does the validity of a local Steiner formula imply convexity?

The positive answer is given in the following theorem.

Theorem 2. Let $A \subset \mathbb{R}^{d}$ be non-empty and closed. Assume that for any measurable bounded function $f: \mathbb{R}^{d} \times S^{d-1} \rightarrow \mathbb{R}$ with compact support there are $c_{1}(f), \ldots, c_{d}(f) \in \mathbb{R}$ such that, for all $t>0$,

$$
\int_{\mathbb{R}^{d} \backslash A} f(p(A, z), u(A, z)) \mathbf{1}\{d(A, z) \leq t\} d z=\sum_{j=1}^{d} c_{j}(f) t^{j} .
$$

Then A is convex.

Theorem 2 is an immediate consequence of a more general result which is stated as Theorem 3 below. Let again $A \subset \mathbb{R}^{d}$ be a closed set. The reach of $A$, denoted by reach $(A)$, is defined as the supremum of all $r>0$ such that the metric projection of $z$ on $A$ is defined for all $z \in \mathbb{R}^{d}$ with $d(A, z)<r$, where $\sup \emptyset:=0$. We say that $A$ has positive reach whenever reach $(A)>0$. Of course, a closed convex set $A$ satisfies reach $(A)=\infty$. An extension of the local Steiner formula (1.3) to sets with positive reach was established by Federer [4], a simpler 
and more general approach was later developed by M. Zähle [19]. To state such a local Steiner formula, let $A \subset \mathbb{R}^{d}$ satisfy $\operatorname{reach}(A) \geq r$. Then, for any measurable bounded function $f: \mathbb{R}^{d} \times S^{d-1} \rightarrow \mathbb{R}$ with compact support and $t \in(0, r)$,

$$
\begin{array}{r}
\int_{\mathbb{R}^{d} \backslash A} f(p(A, z), u(A, z)) \mathbf{1}\{d(A, z) \leq t\} d z \\
=\sum_{j=0}^{d-1} t^{d-j} \kappa_{d-j} \int f(x, u) C_{j}(A ; d(x, u)) .
\end{array}
$$

Here $C_{j}(A ; \cdot), j \in\{0, \ldots, d-1\}$, are locally finite signed measures on the Borel sets of $\mathbb{R}^{d} \times S^{d-1}$ which extend the support measures of convex sets.

The following theorem provides a corresponding characterization of sets with positive reach which generalizes Theorem 2 .

Theorem 3. Let $A \subset \mathbb{R}^{d}$ be non-empty and closed. Assume that for any measurable bounded function $f: \mathbb{R}^{d} \times S^{d-1} \rightarrow \mathbb{R}$ with compact support there are $c_{1}(f), \ldots, c_{d}(f) \in \mathbb{R}$ such that, for all $t \in(0, r)$,

$$
\int_{\mathbb{R}^{d} \backslash A} f(p(A, z), u(A, z)) \mathbf{1}\{d(A, z) \leq t\} d z=\sum_{j=1}^{d} c_{j}(f) t^{j} .
$$

Then $\operatorname{reach}(A) \geq r$.

For the proof of Theorem 1, we will compare the given compact set with its convex hull, provide a result concerning the differentiation of the parallel volumes of a compact set, and (in a sense) exploit the fact that among all rectifiable curves connecting two points precisely the segment has minimal length. The crucial tool for the proof of Theorem 3 is a Steiner-type formula for arbitrary closed subsets of $\mathbb{R}^{d}$ that has recently been developed in [10]. We give a brief introduction to such a formula at the beginning of Section 3.

In the following, the Euclidean ball in $\mathbb{R}^{d}$ with center $a \in \mathbb{R}^{d}$ and radius $r \geq 0$ is denoted by $B^{d}(a, r)$. The $i$-dimensional Hausdorff measure is denoted by $\overline{\mathcal{H}}^{i}$. The interior of a set $A \subset \mathbb{R}^{d}$ is denoted by $\operatorname{int}(A)$.

\section{Proof of Theorem 1}

The two-dimensional special case of the following lemma will play a crucial role in the first part of the proof of Theorem 1 . In the very special case of a compact convex set, a stronger assertion is well known.

Lemma 1. Let $A \subset \mathbb{R}^{d}$ be compact. Then

$$
\frac{d}{d t} V_{d}\left(A_{\oplus t}\right)=\mathcal{H}^{d-1}\left(\partial A_{\oplus t}\right)
$$

for $\mathcal{H}^{1}$-almost all $t>0$. 
Proof. Put $f(x):=d(A, x), x \in \mathbb{R}^{d}$. By Lemma 3.2.34 in [5], we have

$$
\mathcal{H}^{d}\left(A_{\oplus t} \backslash A\right)=\int_{0}^{t} \mathcal{H}^{d-1}\left(f^{-1}(\{s\})\right) d s .
$$

It is easy to check that $\partial A_{\oplus s} \subset f^{-1}(\{s\})$ for all $s>0$. In general, this inclusion cannot be replaced by an equality. Instead, $\mathcal{H}^{d-1}\left(f^{-1}(\{s\}) \backslash \partial A_{\oplus s}\right)=0$ will be shown for $\mathcal{H}^{1}$-almost all $s>0$. Since $\mathcal{H}^{d}(\operatorname{exo}(A))=0$, an application of the coarea formula (see [5]), applied to the distance function $f$, shows that, for $\mathcal{H}^{1}$-almost all $s>0$,

$$
\mathcal{H}^{d-1}\left(\operatorname{exo}(A) \cap f^{-1}(\{s\})\right)=0 .
$$

Let $s>0$ satisfy (2.2) and choose any $x \in f^{-1}(\{s\}) \backslash \operatorname{exo}(A)$. Then $x \in A_{\oplus s}$, and we will show that $x \notin \operatorname{int}\left(A_{\oplus s}\right)$. In fact, since $x \notin \operatorname{exo}(A)$, there is a uniquely determined point $y \in A$ such that $B^{d}(x, s) \cap A=\{y\}$. Hence, for any $z \in$ $A \backslash \operatorname{int}\left(B^{d}(y, s)\right)$, we have $|z-x|>s$. This implies the existence of some $\epsilon>0$ such that $z \notin B^{d}(x, s+\epsilon)$ whenever $z \in A \backslash \operatorname{int}\left(B^{d}(y, s)\right)$. Thus we get

$$
A \subset\left[\mathbb{R}^{d} \backslash B^{d}(x, s+\epsilon)\right] \cup\left[B^{d}(y, s) \backslash \operatorname{int}\left(B^{d}(x, s)\right)\right] .
$$

From (2.3) we infer that $x+\lambda|x-y|^{-1}(x-y) \notin A_{\oplus s}$ for $\lambda \in(0, \epsilon)$.

So far we have shown that $f^{-1}(\{s\})$ can be replaced by $\partial A_{\oplus s}$ under the integral in (2.1). The assertion of the lemma then follows by a well known property of absolutely continuous functions.

Proof of Theorem 1. Let $A \subset \mathbb{R}^{2}$ be a compact and non-empty set satisfying (1.2). We will show that $A$ is equal to its convex hull $C:=\operatorname{conv}(A)$. The proof is divided into three steps.

I. Since $C$ is convex,

$$
V_{2}\left(C_{\oplus t}\right)=V_{2}(C)+d_{1} t+\kappa_{2} t^{2}, \quad t \geq 0,
$$

where $d_{1}$ is the boundary length of $C$. Since there is some $a \in A$ and $A \subset C$, we get $\{a\}_{\oplus t} \subset A_{\oplus t} \subset C_{\oplus t}$, and hence

$$
\kappa_{2} t^{2} \leq V_{2}\left(A_{\oplus t}\right) \leq V_{2}\left(C_{\oplus t}\right) .
$$

A comparison of (1.2), (2.4) and (2.5) first shows that $\kappa_{2} \leq c_{2} \leq \kappa_{2}$, thus $\kappa_{2}=c_{2}$, and consequently $c_{1} \leq d_{1}$. Therefore, for all $t>0$,

$$
\frac{d}{d t} V_{2}\left(A_{\oplus t}\right)=c_{1}+2 \kappa_{2} t \leq d_{1}+2 \kappa_{2} t=\frac{d}{d t} V_{2}\left(C_{\oplus t}\right) .
$$

Combining (2.6) and Lemma 1, we get, for $\mathcal{H}^{1}$-almost all $t>0$,

$$
\mathcal{H}^{1}\left(\partial A_{\oplus t}\right) \leq \mathcal{H}^{1}\left(\partial C_{\oplus t}\right) .
$$


II. We fix $t>\operatorname{diam}(A)$ (the diameter of $A$ ) such that (2.7) is satisfied. Then by Lemma 1 in [3] and after a translation, the set $A_{\oplus t}$ is a star body (see [16]). In particular, $\varphi: S^{1} \rightarrow \partial A_{\oplus t}, u \mapsto \rho\left(A_{\oplus t}, u\right) u$ is a homeomorphism, where $\rho\left(A_{\oplus t}, \cdot\right)$ denotes the radial function of $A_{\oplus t}$. Since $A_{\oplus t} \subset C_{\oplus t}$, we have that $\rho\left(A_{\oplus t}, u\right) \leq \rho\left(C_{\oplus t}, u\right)$ for all $u \in S^{1}$. Now we define the spherical set $\Omega:=\{u \in$ $\left.S^{1}: \rho\left(A_{\oplus t}, u\right)<\rho\left(C_{\oplus t}, u\right)\right\}$.

Since $\Omega$ is an open subset of $S^{1}$, there is an at most countable (possibly empty) family $\mathcal{S}$ of mutually disjoint non-empty open spherical segments $\omega \subset S^{1}$ such that $\Omega=\bigcup_{\omega \in \mathcal{S}} \omega$. Each support line of $C_{\oplus t}$ contains a point of $\partial A_{\oplus t} \cap \partial C_{\oplus t}$. Hence each open halfspace contains some $u \notin \Omega$. Therefore, for all $\omega \in \mathcal{S}$, $\mathcal{H}^{1}(\omega)<\pi$ and $\omega$ can be represented as the open geodesic segment between two points $\alpha_{\omega}$ and $\beta_{\omega}$ in $S^{1}$. Let $\bar{\omega}$ denote the closure of $\omega$.

Let $\psi: S^{1} \rightarrow \partial C_{\oplus t}$ be defined by $\psi(u):=\rho\left(C_{\oplus t}, u\right) u$. Then $a_{\omega}:=\varphi\left(\alpha_{\omega}\right)=$ $\psi\left(\alpha_{\omega}\right)$ and $b_{\omega}:=\varphi\left(\beta_{\omega}\right)=\psi\left(\beta_{\omega}\right)$ for $\omega \in \mathcal{S}$. Next we show that $I_{\omega}:=\psi(\bar{\omega})$ is a segment for $\omega \in \mathcal{S}$. Choose an arbitrary point $\gamma_{\omega} \in \omega$, put $c_{\omega}:=\psi\left(\gamma_{\omega}\right)$, and let $H_{\omega}$ denote a support line of $C_{\oplus t}$ at $c_{\omega}$. It is sufficient to show that $a_{\omega}, b_{\omega} \in H_{\omega}$. Since $C_{\oplus t}=\operatorname{conv}\left(A_{\oplus t}\right)$ and $c_{\omega} \in H_{\omega}$, there are points $a_{\omega}^{\prime}, b_{\omega}^{\prime} \in \partial A_{\oplus t} \cap H_{\omega} \subset \partial C_{\oplus t}$ such that $c_{\omega} \in\left(a_{\omega}^{\prime}, b_{\omega}^{\prime}\right)$, where $a_{\omega}^{\prime} \neq b_{\omega}^{\prime}$ by the choice of $c_{\omega}$. It follows that $a_{\omega}^{\prime}, b_{\omega}^{\prime} \notin \psi(\omega)$ so that $a_{\omega} \in \operatorname{conv}\left(\left\{0, a_{\omega}^{\prime}, b_{\omega}^{\prime}\right\}\right)$. Since $a_{\omega}$ is a boundary point of $C_{\oplus t}$, it lies in $\left[0, a_{\omega}^{\prime}\right) \cup\left[0, b_{\omega}^{\prime}\right)$ or in $\left[a_{\omega}^{\prime}, b_{\omega}^{\prime}\right]$. The first case is clearly impossible as $0 \in \operatorname{int}\left(C_{\oplus t}\right)$. Hence $a_{\omega} \in\left[a_{\omega}^{\prime}, b_{\omega}^{\prime}\right] \subset H_{\omega}$, and by symmetry $b_{\omega} \in H_{\omega}$.

We put $\Gamma_{\omega}:=\varphi(\bar{\omega})$ and next prove that

$$
\mathcal{H}^{1}\left(\Gamma_{\omega}\right) \geq \mathcal{H}^{1}\left(I_{\omega}\right)
$$

for all $\omega \in \mathcal{S}$. Let $H_{\omega}$ denote the line which contains $I_{\omega}$, and let $J_{\omega}$ denote the orthogonal projection of $\Gamma_{\omega}$ onto $H_{\omega}$. Since $a_{\omega}, b_{\omega} \in J_{\omega}$ and $\Gamma_{\omega}$ is (topologically) connected, we get that $I_{\omega} \subset J_{\omega}$. Moreover, since the orthogonal projection onto $H_{\omega}$ is a contraction, we obtain that $\mathcal{H}^{1}\left(\Gamma_{\omega}\right) \geq \mathcal{H}^{1}\left(J_{\omega}\right) \geq \mathcal{H}^{1}\left(I_{\omega}\right)$, i.e. (2.8). Inequality (2.7) implies that

$$
0 \leq \mathcal{H}^{1}\left(\partial C_{\oplus t}\right)-\mathcal{H}^{1}\left(\partial A_{\oplus t}\right)=\sum_{\omega \in \mathcal{S}}\left(\mathcal{H}^{1}\left(I_{\omega}\right)-\mathcal{H}^{1}\left(\Gamma_{\omega}\right)\right) .
$$

From (2.8) and (2.9) we deduce that

$$
\mathcal{H}^{1}\left(\Gamma_{\omega}\right)=\mathcal{H}^{1}\left(I_{\omega}\right)
$$

for $\omega \in \mathcal{S}$. For any $\omega \in \mathcal{S}$, we have $p_{\omega}:=\varphi\left(\gamma_{\omega}\right) \notin I_{\omega}$. Let $\omega^{\prime}$ and $\omega^{\prime \prime}$ denote the geodesic segments connecting $\alpha_{\omega}, \gamma_{\omega}$ and $\gamma_{\omega}, \beta_{\omega}$ in $S^{1}$. By the triangle inequality and by repeating the argument leading to (2.8), we then get

$$
\mathcal{H}^{1}\left(I_{\omega}\right)<\left|p_{\omega}-a_{\omega}\right|+\left|b_{\omega}-p_{\omega}\right| \leq \mathcal{H}^{1}\left(\varphi\left(\omega^{\prime}\right)\right)+\mathcal{H}^{1}\left(\varphi\left(\omega^{\prime \prime}\right)\right)=\mathcal{H}^{1}\left(\Gamma_{\omega}\right),
$$

a contradiction. This implies that $\mathcal{S}=\emptyset$, and therefore $A_{\oplus t}=C_{\oplus t}$ is convex. 
III. So far we have shown that $A_{\oplus t}$ is convex for $\mathcal{H}^{1}$-almost all $t>\operatorname{diam}(A)$. Hence $A_{\oplus r}$ is convex for every $r>\operatorname{diam}(A)$, and thus $A_{\oplus r}=C_{\oplus r}$. In particular, $V_{2}\left(A_{\oplus r}\right)=V_{2}\left(C_{\oplus r}\right)$ for $r>\operatorname{diam}(A)$, and hence by (1.2) and the convexity of $C$,

$$
V_{2}(A)+c_{1} r+\kappa_{2} r^{2}=V_{2}(C)+d_{1} r+\kappa_{2} r^{2},
$$

first for $r>\operatorname{diam}(A)$, but then also for any $r \geq 0$. This in turn shows that $V_{2}\left(A_{\oplus r}\right)=V_{2}\left(C_{\oplus r}\right)$ for $r \geq 0$. Since $A_{\oplus r}$ is compact and $C_{\oplus r}$ is the closure of its interior for $r>0$, we deduce that $A_{\oplus r}=C_{\oplus r}$ is convex for any $r>0$. This implies the asserted convexity of $A$.

In the second part of the proof of Theorem 1, one can use a global integralgeometric Crofton formula instead of arguing locally by distinguishing between different parts of the boundaries of $A_{\oplus t}$ and $C_{\oplus t}$.

\section{Support measures and proof of Theorem 3}

First, we recall the main result of [10]. Let $A \subset \mathbb{R}^{d}$ be a non-empty closed set. The normal bundle of $A$ is defined by

$$
N(A):=\{(p(A, z), u(A, z)): z \notin A \cup \operatorname{exo}(A)\} .
$$

It is a measurable subset of $\partial A \times S^{d-1}$. The reach function $\delta(A, \cdot): \mathbb{R}^{d} \times S^{d-1} \rightarrow$ $[0, \infty]$ of $A$ is defined by

$$
\delta(A, x, u):=\inf \{t \geq 0: x+t u \in \operatorname{exo}(A)\}, \quad(x, u) \in N(A),
$$

and $\delta(A, x, u):=0$ for $(x, u) \notin N(A)$. Note that $\delta(A, \cdot)>0$ on $N(A)$. By Lemma 6.2 in [10], $\delta(A, \cdot)$ is a measurable function. The reach function of $A$ localizes the notion of reach introduced in Section 1 (see also [4]). It is easy to check that $\operatorname{reach}(A)=\inf \{\delta(A, x, u):(x, u) \in N(A)\}$. In particular, $A$ is convex if and only if $\delta(A, \cdot) \equiv \infty$ on $N(A)$.

In [10], the support measures $\mu_{0}(A ; \cdot), \ldots, \mu_{d-1}(A ; \cdot)$ of a non-empty closed set $A \subset \mathbb{R}^{d}$ have been introduced as real-valued functions, defined on all Borel subsets of $\mathbb{R}^{d} \times S^{d-1}$ which are contained in

$$
\left(\left(\mathbb{R}^{d} \times S^{d-1}\right) \backslash N(A)\right) \cup\{(x, u): x \in B, \delta(A, x, u) \geq s\},
$$

for some $s>0$ and some compact $B \subset \mathbb{R}^{d}$. These signed measures vanish on each Borel subset of $\left(\mathbb{R}^{d} \times S^{d-1}\right) \backslash N(A)$. The total variation measure of $\mu_{i}(A ; \cdot)$ is denoted by $\left|\mu_{i}\right|(A ; \cdot)$.

Put $a \wedge b:=\min \{a, b\}$ for $a, b \in \mathbb{R}$. Then Theorem 2.1 in [10] states that

$$
\int_{N(A)} \mathbf{1}\{x \in B\}(\delta(A, x, u) \wedge r)^{d-j}\left|\mu_{j}\right|(A ; d(x, u))<\infty,
$$


$j=0, \ldots, d-1$, for all compact sets $B \subset \mathbb{R}^{d}$ and all $r>0$, and, for any measurable bounded function $f: \mathbb{R}^{d} \rightarrow \mathbb{R}$ with compact support,

$$
\begin{aligned}
\int_{\mathbb{R}^{d} \backslash A} f(z) d z= & \sum_{i=0}^{d-1}(d-i) \kappa_{d-i} \int_{0}^{\infty} \int_{N(A)} t^{d-1-i} \mathbf{1}\{t<\delta(A, x, u)\} \\
& \times f(x+t u) \mu_{i}(A ; d(x, u)) d t .
\end{aligned}
$$

The support measures are uniquely determined by the local Steiner-type formula (3.1).

Proof of Theorem 3. We fix some $\tau \in(0, r)$ and a compact set $B \subset \mathbb{R}^{d}$. Then we put

$$
N_{\tau, B}:=\{(x, u) \in N(A): \delta(A, x, u) \leq \tau, x \in B\} .
$$

Let $f: \mathbb{R}^{d} \times S^{d-1} \rightarrow[0,1]$ be defined by $f(x, u):=\mathbf{1}\left\{(x, u) \in N_{\tau, B}\right\}$. Then (1.4) and (3.1) imply that, for all $t \in(0, r)$,

$$
\begin{aligned}
\sum_{j=1}^{d} c_{j}(f) t^{j}= & \sum_{i=0}^{d-1}(d-i) \kappa_{d-i} \int_{0}^{t} \int_{N_{\tau, B}} s^{d-1-i} \\
& \times \mathbf{1}\{s<\delta(A, x, u)\} \mu_{i}(A ; d(x, u)) d s
\end{aligned}
$$

for some $c_{1}(f), \ldots, c_{d}(f) \in \mathbb{R}$. The function on the right-hand side of (3.2) is independent of $t$ for $t \in(\tau, r)$. Hence the polynomial on the left-hand side of (3.2) must be zero, which implies that

$$
\mathcal{H}^{d}\left(\left\{z \in A_{\oplus r} \backslash A:(p(A, z), u(A, z)) \in N_{\tau, B}\right\}\right)=0 .
$$

Since this is true for any $\tau \in(0, r)$ and any compact set $B \subset \mathbb{R}^{d}$, we obtain that $\delta(A, p(A, z), u(A, z)) \geq r$ for $\mathcal{H}^{d}$-almost all $z \in A_{\oplus r} \backslash A$. We will show that this implies that $\operatorname{reach}(A) \geq r$.

Let $z \in \mathbb{R}^{d} \backslash A$ with $r_{0}:=d(A, z)<r$. There is a sequence of points $z_{i} \in A_{\oplus r} \backslash A, i \in \mathbb{N}$, converging to $z$ as $i \rightarrow \infty$ for which the metric projection $p_{i}:=p\left(A, z_{i}\right) \in A$ exists and $\delta\left(A, p_{i}, u_{i}\right) \geq r$ with $u_{i}:=u\left(A, z_{i}\right) \in$ $S^{d-1}$. Passing to a subsequence and changing notation (if necessary) we can assume that $p_{i} \rightarrow p \in A$ and $u_{i} \rightarrow u \in S^{d-1}$. Choose $r_{1} \in\left(r_{0}, r\right)$. Then $B^{d}\left(p_{i}+r_{1} u_{i}, r_{1}\right) \cap A=\left\{p_{i}\right\}$ for all $i \in \mathbb{N}$; hence $A$ does not intersect the interior of $B^{d}\left(p+r_{1} u, r_{1}\right)$. Clearly, $z_{i}=p_{i}+d\left(A, z_{i}\right) u_{i}$ for $i \in \mathbb{N}$, and therefore $z=p+d(A, z) u=p+r_{0} u$. Moreover, $B^{d}\left(p+r_{0} u, r_{0}\right) \cap A=\{p\}$, which shows that $p$ is the unique nearest point of $z$ in $A$. This proves the required assertion. 


\section{Examples}

This section is devoted to the construction of non-convex, compact sets in $\mathbb{R}^{d}$, $d \geq 3$, whose parallel volumes are polynomials. For this purpose let $L$ be a $(d-2)$-dimensional linear subspace of $\mathbb{R}^{d}$, and let $C \subset L$ be a $(d-2)$-dimensional compact convex set. Then fix a closed set $\hat{A} \subset C$ with $\partial_{L} C \subset \hat{A}$, where $\partial_{L} C$ denotes the boundary of $C$ relative to $L$. Then, for $t \geq \operatorname{diam}(C) / 2$, we will show that the set $A:=\hat{A}_{\oplus t} \subset \mathbb{R}^{d}$ has polynomial parallel volume, i.e.

$$
V_{d}\left(A_{\oplus r}\right)=\sum_{k=0}^{d} c_{k} r^{k}, \quad r \geq 0,
$$

where $c_{k} \in \mathbb{R}$ for $k=0, \ldots, d$. The set $A$ is convex if and only if $\hat{A}$ is convex.

Since $A_{\oplus r}=\hat{A}_{\oplus(t+r)}$, we deduce that

$$
V_{d}\left(A_{\oplus r}\right)=V_{d}\left(C_{\oplus(t+r)}\right)-V_{d}\left(C_{\oplus(t+r)} \backslash \hat{A}_{\oplus(t+r)}\right),
$$

where $V_{d}\left(C_{\oplus(t+r)}\right)$ is a polynomial in $r \geq 0$ due to the convexity of $C$. As we will now see, the second term in the sum does not depend on $r \geq 0$, but is in fact a constant.

For any $y \in L$,

$$
\hat{A}_{\oplus(t+r)} \cap\left(y+L^{\perp}\right)= \begin{cases}y+B_{L^{\perp}}\left(\sqrt{(t+r)^{2}-d(\hat{A}, y)^{2}}\right), & \text { if } d(\hat{A}, y) \leq t+r, \\ \emptyset, & \text { otherwise, }\end{cases}
$$

where $B_{L^{\perp}}(s)=B^{d}(0, s) \cap L^{\perp}$ denotes the ball in $L^{\perp}$ with center 0 and radius $s$. This equation also holds with $\hat{A}$ replaced by $C$. Moreover, since $\partial_{L} C \subset \hat{A}$, we get that $d(\hat{A}, y)=d(C, y)$ whenever $y \in L \backslash C$, and thus

$$
\hat{A}_{\oplus(t+r)} \cap\left((L \backslash C) \times L^{\perp}\right)=C_{\oplus(t+r)} \cap\left((L \backslash C) \times L^{\perp}\right) .
$$

Therefore,

$$
\begin{aligned}
& V_{d}\left(C_{\oplus(t+r)} \backslash \hat{A}_{\oplus(t+r)}\right) \\
& =V_{d}\left(C_{\oplus(t+r)}\right)-V_{d}\left(\hat{A}_{\oplus(t+r)}\right) \\
& =\int_{C} \int_{L^{\perp}}\left(\mathbf{1}\left\{y+z \in C_{\oplus(t+r)}\right\}-\mathbf{1}\left\{y+z \in \hat{A}_{\oplus(t+r)}\right\}\right) \mathcal{H}^{2}(d z) \mathcal{H}^{d-2}(d y),
\end{aligned}
$$

where Fubini's theorem and (4.1) were applied in the last step. Since $t \geq$ $\operatorname{diam}(C) / 2$, and hence $d(\hat{A}, y) \leq t+r$ for $r \geq 0$ and $y \in C$, we further deduce that

$$
\begin{aligned}
& V_{d}\left(C_{\oplus(t+r)} \backslash \hat{A}_{\oplus(t+r)}\right) \\
& =\int_{C} \mathcal{H}^{2}\left(B_{L^{\perp}}(t+r)\right)-\mathcal{H}^{2}\left(B_{L^{\perp}}\left(\sqrt{(t+r)^{2}-d(\hat{A}, y)^{2}}\right)\right) \mathcal{H}^{d-2}(d y) \\
& =\pi \int_{L} d(\hat{A}, y)^{2} \mathcal{H}^{d-2}(d y),
\end{aligned}
$$


which is independent of $r \geq 0$. Thus, $V_{d}\left(A_{\oplus r}\right)$ is a polynomial in $r \geq 0$ with non-negative coefficients.

Turning our attention to special cases, in dimension $d=3$ for instance, $C$ can be a segment of length 2 and $\hat{A}$ the set consisting of the two endpoints of $C$. For $t=1$, the set $A$ is then the union of two touching balls with radius 1 .

More generally, let $\hat{A} \subset L$ be a compact set whose convex hull is a ball in $L$ with radius $s$ and take $A:=\hat{A}_{\oplus t}$ for some $t \geq s$. (In dimension $d=3, \hat{A}$ can be any compact subset of a line.) Then the parallel volume of $A$ is a polynomial. However, if $\hat{A}$ is non-convex, then $A$ is non-convex as well.

If $A \subset \mathbb{R}^{d}$ is any one of the sets constructed above, if $n$ is an even natural number, and if $\mathbb{R}^{d}$ is identified with a $d$-dimensional linear subspace of $\mathbb{R}^{d+n}$, then the parallel volume of $A$ in $\mathbb{R}^{d+n}$ is again a polynomial.

\section{References}

1. Aleksandrov, A.D.: Zur Theorie der gemischten Volumina von konvexen Körpern, I. Verallgemeinerung einiger Begriffe der Theorie der konvexen Körper (in Russian). Mat. Sbornik N.S. 2, 947-972 (1937)

2. Betke, U., Henk, M., Wills, J.M.: Finite and infinite packings. J. Reine Angew. Math. 453, 165-191 (1994)

3. Brown, M.: Sets of constant distance from a planar set. Michigan Math. J. 19, 321-323 (1972)

4. Federer, H.: Curvature measures. Trans. Amer. Math. Soc. 93, 418-491 (1959)

5. Federer, H.: Geometric Measure Theory. Springer, Berlin, 1969

6. Fenchel, W., Jessen, B.: Mengenfunktionen und konvexe Körper. Danske Vid. Selsk. Mat.Fys. Medd. 16, 1-31 (1938)

7. Giannopoulos, A.A., Milman, V.D.: Euclidean structure in finite dimensional normed spaces, 707-779, W. B. Johnson, J. Lindenstrauss (Eds.), Handbook of the Geometry of Banach Spaces. Volume 1, Elsevier, Amsterdam, 2001

8. Hadwiger, H.: Über das Volumen der Parallelmengen, Mitt. Naturf. Ges. Bern 3, 121-125 (1946)

9. Hotelling, H.: Tubes and spheres in $n$-space, and a class of statistical problems. Amer. J. Math. 61, 440-460 (1939)

10. Hug, D., Last, G., Weil, W.: A local Steiner-type formula for general closed sets and applications. Math. Z. 246, 237-272 (2004)

11. Kuriki, S., Takemura, A.: Tail probabilities of the maxima of multilinear forms and their applications. Ann. Stat. 29, 328-371 (2001)

12. Mecke, K.R., Stoyan, D.: (Eds.), Statistical Physics and Spatial Statistics - The Art of Analyzing and Modeling Spatial Structures and Pattern Formation. Lecture Notes in Physics 554, Springer-Verlag, Berlin, Heidelberg, New York, 2000

13. Mecke, K.R., Stoyan, D.: (Eds.), Morphology of Condensed Matter - Physics and Geometry of Spatially Complex Systems. Lecture Notes in Physics 600, Springer-Verlag, Berlin, Heidelberg, New York, 2002

14. Schneider, R.: Bestimmung konvexer Körper durch Krümmungsmaße. Comment. Math. Helv. 54, 42-60 (1979)

15. Schneider, R.: Parallelmengen mit Vielfachheit und Steiner-Formeln. Geom. Dedicata 9, 111-127 (1980) 
16. Schneider, R.: Convex Bodies: the Brunn-Minkowski Theory. Encyclopedia of Mathematics and its Applications 44, Cambridge University Press, Cambridge, 1993

17. Schneider, R., Weil, W.: Stochastische Geometrie. Teubner Skripten zur Mathematischen Stochastik, Teubner, Stuttgart, 2000

18. Stoyan, D., Kendall, W.S., Mecke, J.: Stochastic Geometry and its Applications. Second Edition, Wiley, Chichester, 1995

19. Zähle, M.: Integral and current representation of Federer's curvature measures. Arch. Math. 46, 557-567 (1986) 